\title{
DISCOVERING EVERYDAY MATHEMATICAL SITUATIONS OUTSIDE THE CLASSROOM WITH MATHCITYMAP AND GEOGEBRA 3D
}

\author{
Zsolt Lavicza ${ }^{1}$, Ben Haas ${ }^{1}$ and Yves Kreis ${ }^{2}$ \\ ${ }^{1}$ Johannes Kepler University, Linz School of Education, Austria \\ 2 University of Luxemburg, Luxemburg
}

\begin{abstract}
In elementary school, teaching and learning activities aim to develop, among others, students' skills to acquire deeper understanding of their living environments. There are numerous opportunities for students to recognize forms, shapes, and mathematical connections in everyday situations. These everyday situations can be simulated in classrooms; however, educational technologies offer new approaches to extend classroom activities, teachers can simulate and design shapes through Augmented Reality and 3D printing within or beyond the classroom. To stimulate students' everyday mathematical connections utilizing these technologies could assist in developing activities outside the classroom in urban or in natural environments. Through this approach students could utilize or enhance their mathematical and technical skills within their usual living environments. Utilising educational software such as MathCityMap, GeoGebra 3D Calculator, and other 3D modelling software we developed examples of tasks that could offer easy transitions from in- to out-side of classrooms. In this paper, we will describe learning and teaching aims of these tasks and outline further research and development directions to broaden opportunities to develop students' mathematical, design and modelling skills.
\end{abstract}

Keywords: elementary school, geometry, educational technology, outside, mathematical thinking, trail

\section{INTRODUCTION}

In elementary school, numerous curricular topics are strongly related to students' everyday environments and lives. Hence, teachers recurrently attempt to integrate activities into their teaching encouraging students to explore mathematics in their surroundings and their experiment with their mathematical skills to better understand the world around themselves. In addition, new technologies offer new opportunities for such attempts and enable teachers to design challenging and relevant tasks for students. Several teachers, in Luxembourg, become committed to these opportunities and began using technologies to simulate forms, shapes, and mathematical processes in their classrooms. In these classes, students learned to apply content- and process-related skills in mathematical thinking (Selter \& Zannetin, 2018) through the given tasks. Augmented reality was one of the novel technologies utilized to foster students' deeper understanding of forms and shapes (Berdik 2017; González, 2015). In parallel, physical modelling with 3D printing could make students to apply relevant mathematical ideas and interact with real-world problems (Carreira \& Baioa, 2015). Nevertheless, research clearly shows that teachers play a crucial role in the teaching and learning processes and utilising these technologies could guide students' evolution from personal to mathematical meanings (Lieban \& Lavicza, 2019a; 2019b). Students' living environments and experiences obviously are not limited into their schools and classrooms. They live in urban and natural environments where they daily encounter mathematical ideas and patterns, interact with them consciously or unconsciously. For example, commonly used personal objects and architecture surrounding them entail diverse geometric shapes and various mathematical connections,

Lavicza, Z., Haas, B. \& Kreis, Y. (2020). Discovering Everyday Mathematical Situations Outside the Classroom with MathCityMap and GeoGebra 3D. In M. Ludwig, S. Jablonski, A. Caldeira, \& A. Moura (Eds.), Research on Outdoor STEM Education in the digiTal Age.

Proceedings of the ROSETA Online Conference in June 2020 (pp.23-30) Münster: WTM. https://doi.org/10.37626/GA9783959871440.0.03 
orienting themselves to and from schools require spatial navigation skills, and building a hut with their friends in the forest necessitates hidden mathematical ideas to be utilised. Skills and knowledge, they learned in classrooms could be applied beyond school, but most often these connections are not recognised and mathematics considered out of reach from real life. Thus, if education aims to utilise out-of-classroom environments for teaching and learning, these situations would need to be stimulated or scaffolded making them apparent for students. This raises the question of how we could transfer classroom activities outdoors, into students' living environments and offer meaningful and inspiring mathematical tasks to enable new forms of mathematical learning. One of the approaches for such transformations of mathematics to outdoors is the idea of mathematical trails with the utilisation of technologies through a novel educational tool MathCityMap (Ludwig \& Jesberg, 2015). In MathCityMap (MCM) teachers can define trails and create tasks suited to their students in their own environments. Furthermore, MCM also allow creators to design tasks with additional technologies, in our case, augmented reality (AR) and 3D printing, which was already trialled in classroom conditions and then could be utilised in MCM trails. In this paper, we will present examples of tasks based on augmented reality applications within the GeoGebra 3D Calculator, attached to MCM trails as well as possible applications of 3D printing associated with these tasks. We will describe initial tasks and discuss their potentials for further research and developments of theoretical approaches. Furthermore, we aim to develop opportunities to integrate MCM and GeoGebra 3D at a deeper level and internally connect this software for future benefits in outdoor task designs.

\section{CONTEXT, DATA AND TASK DESIGNS}

Creating tasks with augmented reality in the dynamic geometry software GeoGebra 3D Calculator has been part of recent studies we undertook (Lieban \& Lavicza, 2019a; Haas, Kreis \& Lavicza, in review). Based on our experiences, students are experiencing difficulties in the process of visualizing three-dimensional geometric shapes and offering support only in 2D showed similar problems in other studies (Ng, 2017; González, 2015). Neverheless, for our study we decided to experiment with designing tasks that are fully utilising augmented reality applications. Using AR features of the GeoGebra 3D calculator on phones and tablets, students could have opportunities to discover properties of their surroundings real-time and explore as well as modulate these shapes for their own needs. Through these activities we expected that students could foster their visual-spatial memories and visualisation abilities, which are well described in neuroscience research by Szucs et al., (2013). Based on the curricular requirements in Luxembourg (MENJE, 2011) and following the four basic principles of Dienes' theory of mathematics (Hirstein, 2008; Lieban \& Lavicza, 2019b), as Dienes' approach fitted well for our tasks in previous 3D-related studies, we reproduced geometric shapes from students learning environments with AR in GeoGebra 3D. Initially students worked with pre-designed worksheets that they could modulate the shapes fitting real-world objects (Figure 1), but when they became more proficient with the tools, they were able to develop their own modelling applications (Figure 2). 


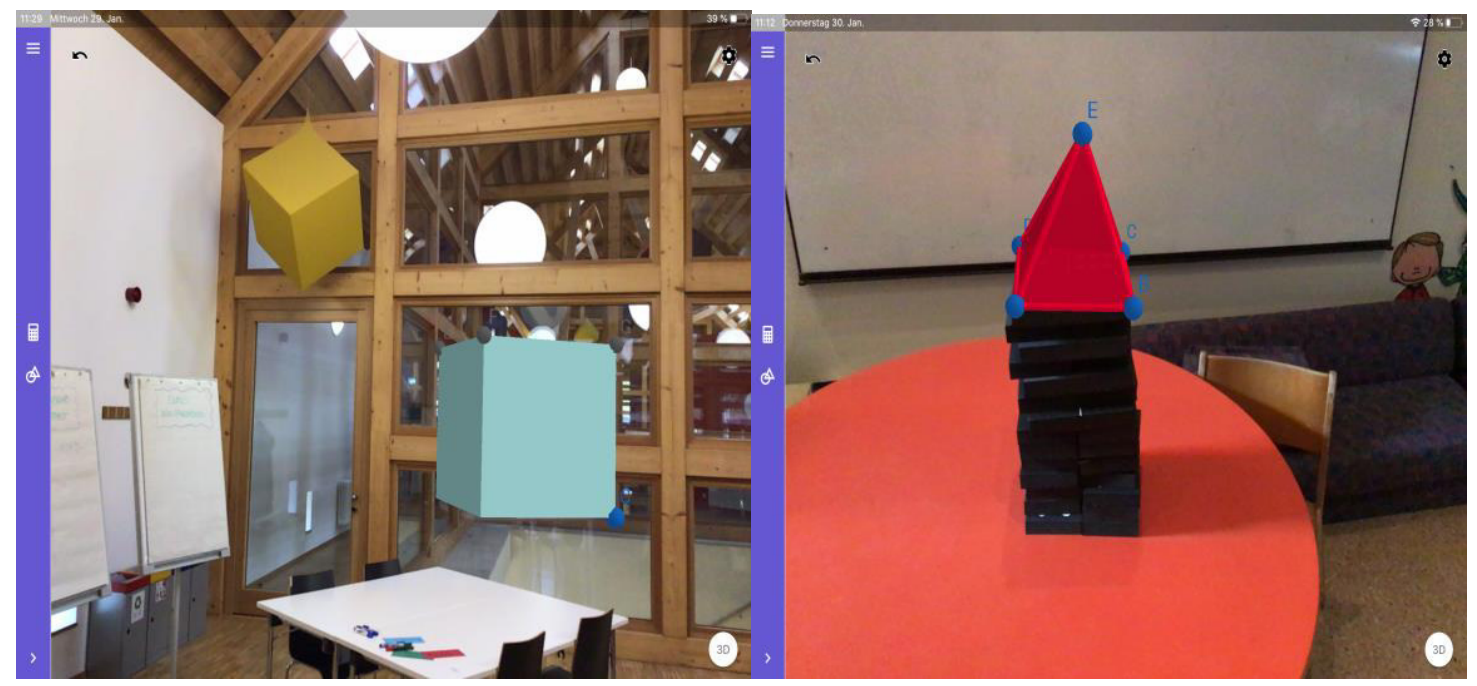

Figure 1: Reproduction of shapes with GeoGebra 3D Calculator.

To further engage the pupils in a deeper modulation process, we worked with them to recreate shapes with the augmented reality functions, objects, composed of different shapes. Figure 2 shows an example in which students created modelling of a paper plan with exact measurements in the software.

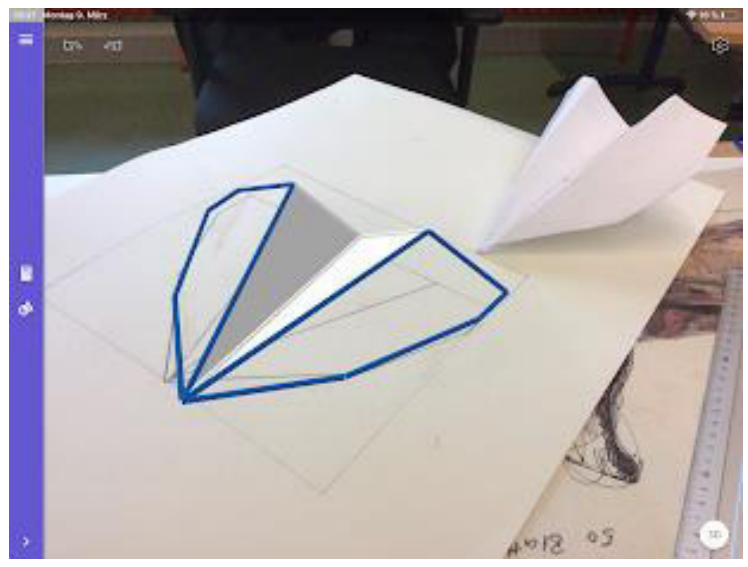

Figure 2: Recreation complex objects with GeoGebra 3D Calculator.

During the execution of different tasks, students were utilising their already learned knowledge in mathematics, software and design as well as their skills in exploration, modelling, and cooperation.

Within the experiments we collected various data from students and teachers. We carried out semi-structured interviews with students in two classes, four Grade-4 teachers, three Kindergarten teachers and a School Principal. In addition, we observed students' activities in school and partly on MCM trails as well as we could collect students' solutions. However, due to the sudden introduction of school closure and confinements due to the COVID-19 emergency, we could not continue working with students in school. Nonetheless, we worked on other AR and 3D Printing online activities that will be recorded in future papers (e.g. Haas, Kreis \& Lavicza, in review). In this paper, we mainly report on the activities we carried out with some preliminary findings and will continue these experiments after schools reopen. 
In the modelling of the previous two examples (Figure 1,2), for some of the groups we observed their uses of the software and recorded their arguments. Further analysis of these records will outline students' design arguments, their uses of mathematical language, descriptions of measurements, and explanations of lengths, areas and volumes. Overall, students' arguments of modelling were containing valid expressions of their mathematical knowledge and development of concepts, but we noticed that further scaffolding of tasks and then later discussion of modelling together with teachers are essential for future designs of such tasks.

The next step of our task design was to integrate our experiences from the initial tasks within the school and move learning to the outside. Thus, we attempted to connect the AR features of GeoGebra 3D Calculator and MCM. As GeoGebra 3D Calculator is available on iPads, we decided to initially utilise this setup and allow students to explore geometric modelling around their schools and at home. Both applications were installed on iPads and could be connected to enable students to explore their environments.

In this experiment, we chose three tasks that we wanted to add into trails in MCM:

- Discovering shapes in the environment (task on cubes)

- Reproducing more complex objects constructed from different geometric shapes (task on woodhouse)

- Designing complex objects created from different shapes (task on the barn gate)

We decided to create each task near a smaller district in our town, where most students from the school live. Thus, we designed the three tasks with GeoGebra 3D Calculator and MCM to engage students with mathematics outside of their classrooms and still benefit from interactions with each other and the AR tool. The tasks we integrated into a trail (Figure 3) and students' designs and solutions had to be posted on our dedicated online portal.

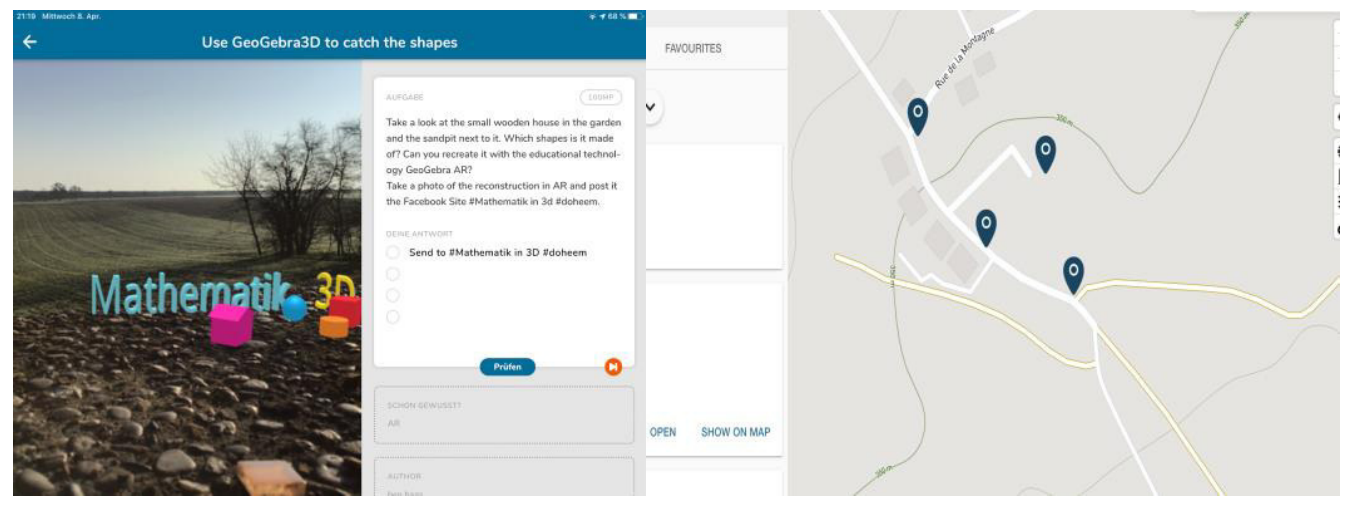

Figure 3: Task presentation in MCM.

In the first task, we wanted students to discover cubes in their environments. This task was similar to some of the exercises on which students had already worked in class. The main aim of this task was to prepare students for forthcoming tasks, further develop their software skills, and follow the different development stages described by Dienes (Hirstein, 2008). 


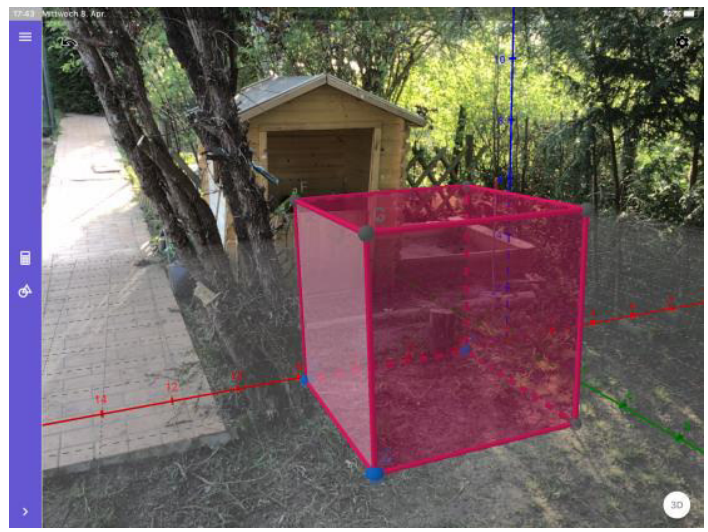

Figure 4: Discovering of shapes in the environment (base of the house).

In the second task, students needed to reconstruct a complex object made from different shapes; for instance, a small wooden house and a sandpit (Figure 5). Firstly, students had to identify the different shapes from which the object was made of. Then, secondly, they had to construct them one-by-one and then join them together on their iPad. To be able to develop a correct construction, they needed to recognise the different properties of shapes, create line segments, and modulate their constructions. Students employed process-related skills, such as modelling and argumentation to be able to solve this task. They also had the opportunity to discuss their tasks with each other and then compare solutions in school together with their teachers.

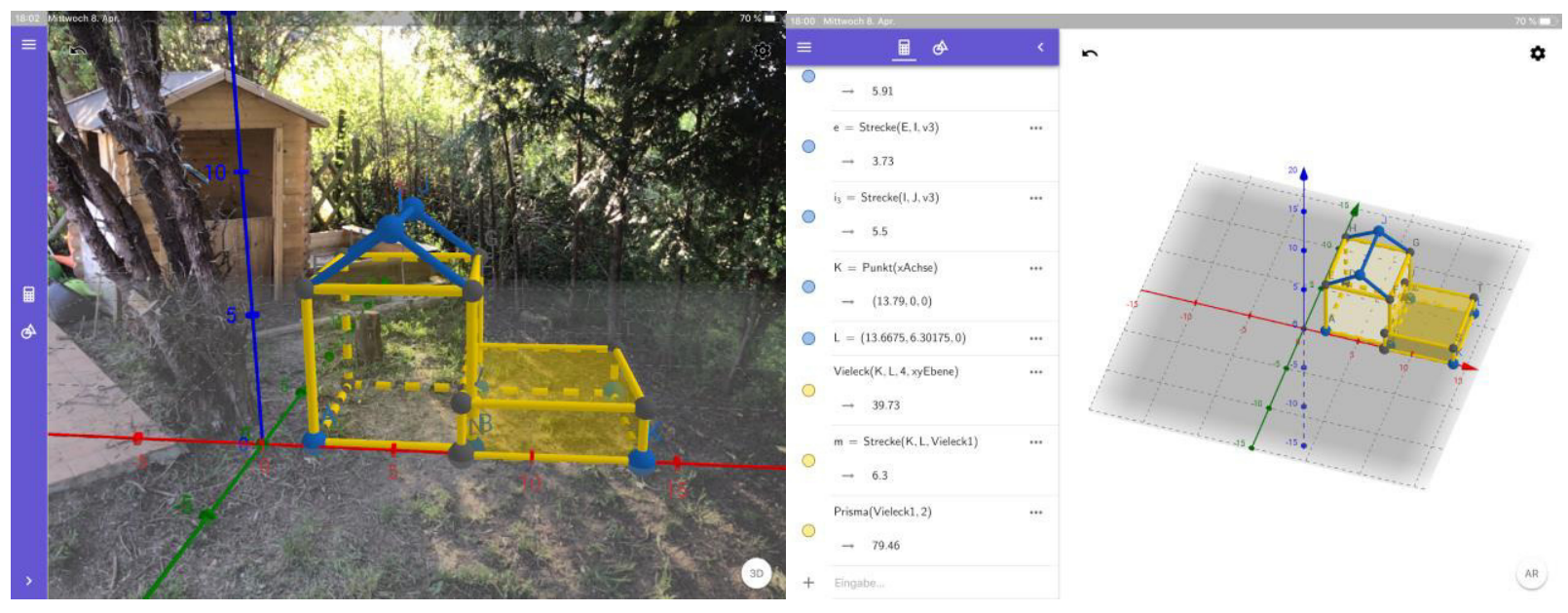

Figure 5: Reconstruction of a complex object made of different shapes in MCM.

In the third task, students needed to create a gate for a barn in 3D GeoGebra Calculator (Figure 6). Students had to identify shapes required for the construction in augmented reality. They had to adapt the size of the shapes, combine them with different shapes, and modulate their construction, so that the missing gate would fit to its place. Once they finished, they posted their results and received feedback both online and in class. 


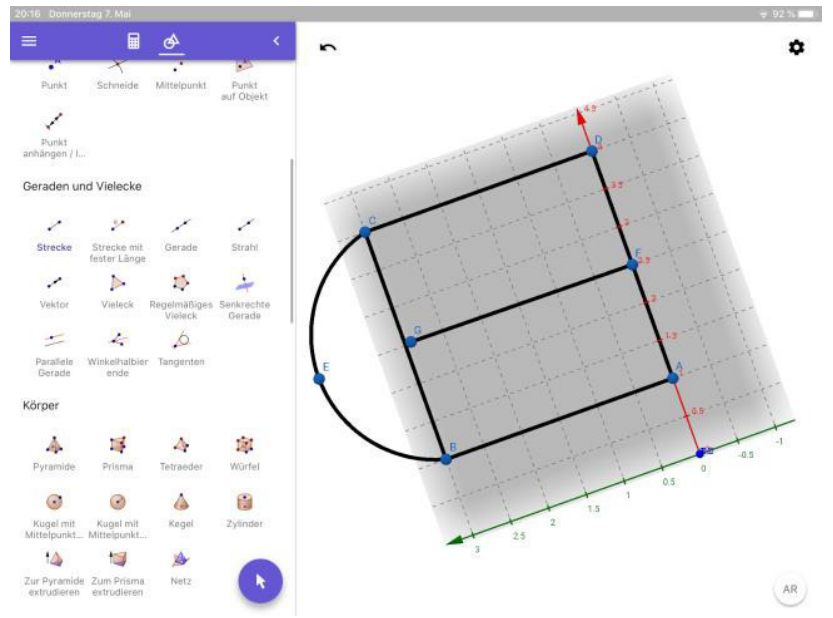

Figure 6: Designing a barn gate and the creation of a complex object.

The tasks above required students to recreate objects with digital modelling through utilising their mathematical and software knowledge. However, we also aimed to inspire students to use these skills to create objects meaningful and useful for their lives and environments. One of the submitted examples came from a trail close to a garden and the head of the watering can was broken next to the newly planted potatoes. Students wrote in their report that they wanted to help, took measurements with AR and designed the watering head with the AR software, using trial and error method, and then 3D printed several prototypes until they fixed the problem (Figure 7). According to students, this was one of the most inspiring part of their mathematics trail. These kinds of problems could be integrated into MCM trails, but possibly open-ended tasks encouraging students to be aware of their surroundings could be explored.

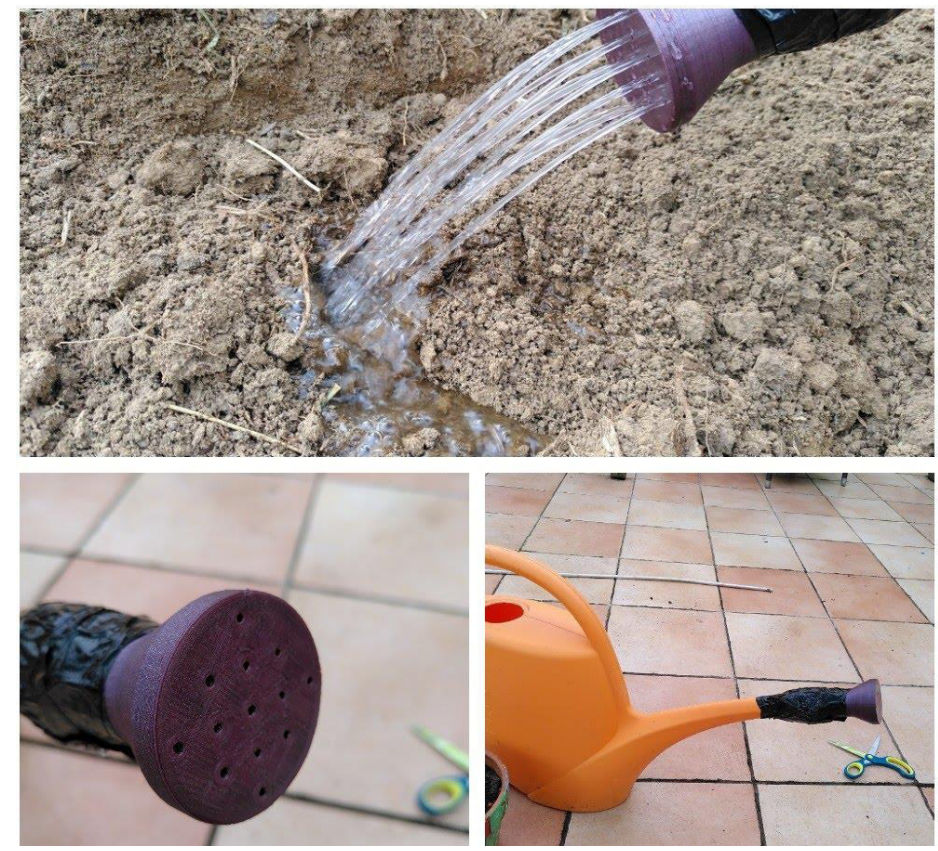

Figure 7: Fixing a watering can with 3D printing.

The gathered interview and reporting data from students and teachers suggest that 3D printing adds one more layer to the understanding of the correctness of students' solutions. 
With these prints students acquire not only visual, but also concrete feedback after touching and observing their printed solutions. Through AR tools they can perform digital modelling and can evaluate their correctness, but assessing results from 3D prints, which also require additional mathematical and software skills, they could immediately recognise if their measurements calculations and modelling approach was correct or not. If some of the elements were incorrect, they would need to repeat modelling until they obtain their desired solution. We believe that this process cycle could be offer opportunities to offer a glance for scientific research methods and engineering processes.

Finally, an interesting solution was submitted by a student who created a castle, he had seen during their travelling, called it "the fortress and the blue magic tower" (Figure 8) and also used this model for his story in a language class on storytelling.

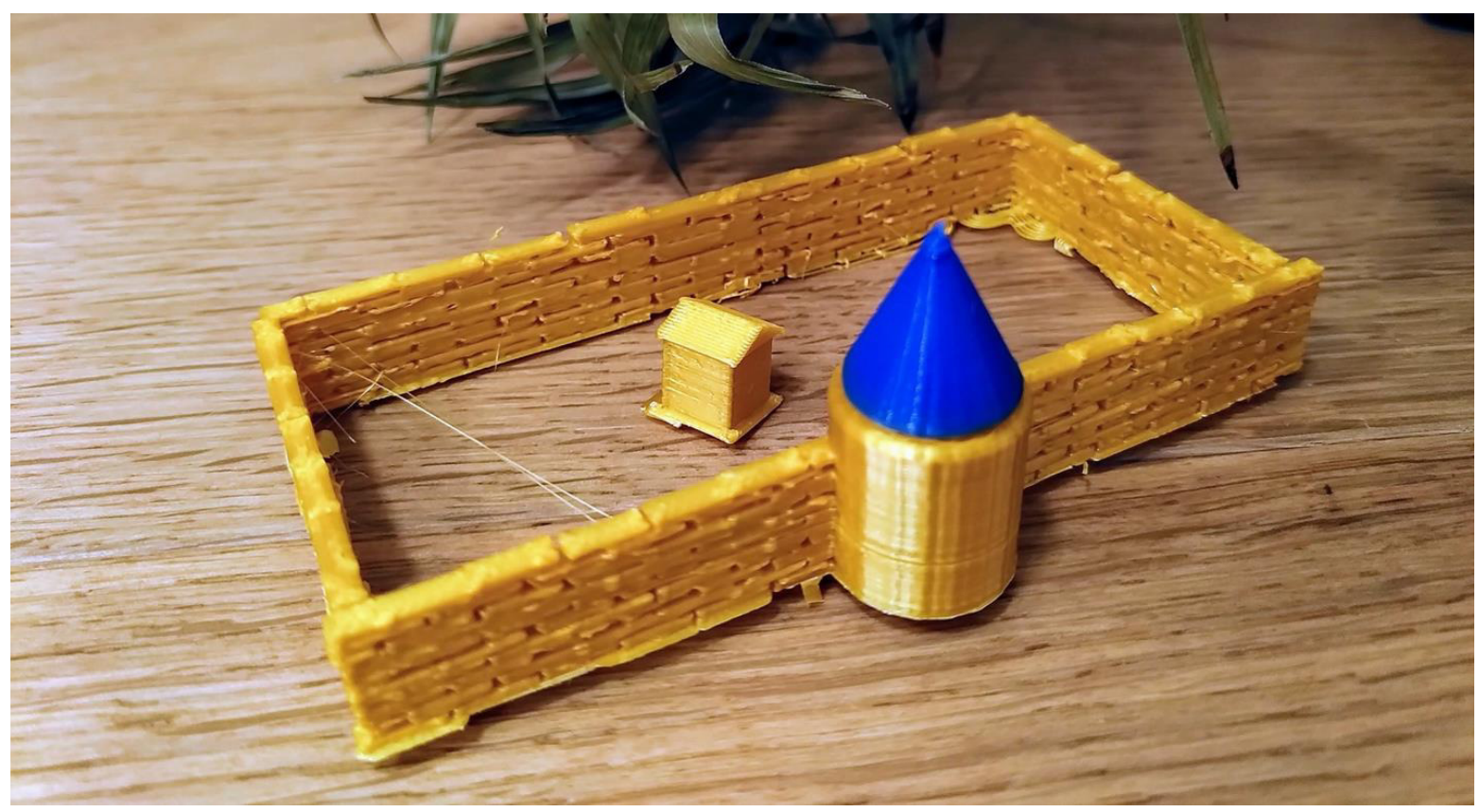

Figure 8: the fortress and the blue magic tower.

Throughout these tasks students acquired variety of knowledge in mathematics, technology and related subjects as well as they become more aware of the mathematical nature of their surroundings, demystifying some previously held beliefs about the subject.

\section{DISCUSSIONS}

Combining the AR features in the GeoGebra 3D Calculator with MCM offered us new opportunities to experiment with novel mathematical approaches beyond classrooms. Teachers can create trails to let the students explore their living environments and make them discover these places with mathematical perspectives. The examples presented in this paper could assist teachers to create new kinds of activities and trails to make mathematics more integrated into students' lives and contribute to reducing the perceived disconnection of mathematics from the real world. Our preliminary data analyses, together with our previous research, showed some potentials for both teachers and students in connecting physical and digital worlds; making mathematical connections with real-world situations; bringing mathematics outside of classrooms; and integrating new technologies 
to create meaningful mathematical tasks. With our examples we explored opportunities to further integrate the two applied mathematical tools GeoGebra 3D and MathCityMap. Designing software integration could potentially offer an even more powerful tool for mathematics teaching in the outside. Certainly, there is a need for more extensive future research in this area, but in this paper, we wanted to showcase some initial opportunities with some tools and approaches that could offer new directions for mathematics teaching and learning.

\section{References}

Berdik, C. (2017). Kids Code Their Own 3D Creations with New Blocks-Based Design Program. Tech Directions, 76(9), 23-24.

Carreira, S., \& Baioa, A. M. (2015). Assessing the best staircase: Students' modeling based on experimentation with real objects. In Proceedings of the Ninth Congress of the European Society for Research in Mathematics Education (pp. 834-840). Prague, Czech Republic.

González, N. A. A. (2015). How to include augmented reality in descriptive geometry teaching. Procedia Computer Science, 75, 250-256.

Haas, B., Kreis, Y., \& Lavicza, Z. (in review). Parent's perspectives: The use of Augmented Reality, digital and physical modelling in remote teaching in elementary school Educational Studies in Mathematics.

Hirstein, J. (2008). The impact of Zoltan Dienes on mathematics teaching in the United States. In B. Sriraman \& Z. P. Dienes (Eds.), Mathematics Education and the Legacy of Zoltan Paul Dienes (pp. 107-111). Charlotte, NC: Information Age Publishing, Inc.

Lieban, D., \& Lavicza, Z. (2019a). Dissecting a Cube as a Teaching Strategy for Enhancing Students' Spatial Reasoning: Combining Physical and Digital Resources. In Bridges 2019 Conference Proceedings, 319-326.

Lieban, D., \& Lavicza, Z. (2019b). Instrumental genesis and heuristic strategies as frameworks to geometric modeling in connecting physical and digital environments. In Eleventh Congress of the European Society for Research in Mathematics Education.

Lieban, D., Ulbrich, E., Barreto, M., \& Lavicza, Z. (2019). A new era of manipulatives: Making your own resources with 3D printing and other technologies. In Eleventh Congress of the European Society for Research in Mathematics Education.

Ludwig, M., \& Jesberg, J. (2015). Using Mobile Technology to Provide Outdoor Modelling Tasks - The MathCityMap-Project. Procedia - Social and Behavioral Sciences, 191, 27762781.

MENJE (2011). Plan d'études, école fondamentale, numéro du courrier de l'éducation nationale spéciale.

Ng, O. (2017). Exploring the use of 3D Computer-Aided Design and 3D Printing for STEAM Learning in Mathematics. Digit Exp Math Educ 3, 257-263, https://doiorg.proxy.bnl.lu/10.1007/s40751-017-0036-x

Selter, C \& Zannetin E. (2018). Mathematik unterrichten in der Grundschule. Seelze: Friedrich Verlag

Szucs, D., Devine, A., Soltesz, F., Nobes, A., \& Gabriel, F. (2013). Developmental dyscalculia is related to visuo-spatial memory and inhibition impairment. Cortex, 49(10), 2674-2688. doi: 10.1016/j.cortex.2013.06.007 